\title{
Correlates of genetic attributions among parents of children in the USA with developmental disabilities
}

Bridget Kiely

Sujit Vettam

Andrew Adesman

Division of Developmental and Behavioral Pediatrics, Department of Pediatrics, Steven and Alexandra Cohen Children's Medical Center of New York, New Hyde Park, NY, USA

Correspondence: Andrew Adesman Division of Developmental and Behavioral Pediatrics, Department of Pediatrics, Steven and Alexandra Cohen Children's Medical Center of New York, 1983 Marcus Avenue, Suite I30, Lake Success, New York II 042, NY, USA

Tel+I 5I68026I0I

Email aadesman@northwell.edu
Introduction: As technologies for identifying causal genetic variants in children with autism spectrum disorders (ASD) and other developmental conditions continue to advance, there is a need to understand the factors that influence parental beliefs about the causes of their child's disabilities. This study assessed the correlates of etiologic attributions among US parents of children with ASD, intellectual disability (ID), and/or developmental delay (DD).

Methods: Data were obtained from the Centers for Disease Control and Prevention's nationally representative Survey of Pathways to Diagnosis and Services. Respondents were classified according to whether their child had ASD without ID or DD (ASD-only), ASD with ID and/or DD (ASD+ID/DD), or ID and/or DD without ASD (ID/DD-only). Respondents rated the extent to which they believed that genetics/heredity and environmental exposures (prenatal and/or postnatal) had contributed to their child's condition. Logistic regression analyses and chi-square tests were used to assess the relationship between parental beliefs and child characteristics.

Results: The parents of children with comorbid ASD and ID/DD were found to be significantly less likely than those in the other condition groups to attribute their child's condition to genetics. Within the ASD+ID/DD group, parental endorsement of genetics was lower among those who reported a history of language regression $(p=0.006)$.

Conclusion: Further research is needed to evaluate the impact of parental genetic attributions on medical decision-making.

Keywords: parental beliefs, etiology, developmental disabilities, autism spectrum disorders, intellectual disability

\section{Introduction}

The rising prevalence of autism spectrum disorders (ASD) in the USA has fueled scientific and popular interest in the causes of this condition. ${ }^{1}$ As a growing body of research continues to reveal the genetic heterogeneity underlying the autism spectrum, clinical genetics professionals are likely to play an increasingly important role in the medical assessment of affected children. ${ }^{2,3}$ In recognition of the potential benefits of obtaining a genetic diagnosis, the American College of Medical Genetics and Genomics has recommended that genetic testing be offered to all children with ASD. ${ }^{4}$ However, genetics services remain underutilized in this population, and recent studies suggest that less than half of all children with ASD undergo recommended genetic testing. ${ }^{5,6}$ Barriers to the utilization of genetics services in this population include limited parental awareness of genetic testing options, concerns about cost, and uncertainty about the value of a genetic diagnosis. ${ }^{6,7}$ 
Although clinicians play a vital role in facilitating access to genetics services for children with ASD, decisions about whether to pursue genetic testing ultimately rest with the parents themselves, who may act on physician recommendations and interpret the results of genetic testing in the context of their existing beliefs about ASD etiology. ${ }^{7,8}$ Studies of parental etiological attributions have produced varying estimates of the proportion of parents that believe their child's ASD has a genetic basis; a 2006 survey of 41 parents found that more than $90 \%$ believed that genetic influences had contributed to their child's ASD, while another study from the same year reported that just $26 \%$ of their 62 -parent sample endorsed genetic predisposition as a cause. ${ }^{9,10}$ These studies have also documented the wide range of nongenetic causes to which parents commonly attribute their child's ASD, including prenatal exposures, perinatal events, vaccinations, dietary sensitivities, child illnesses, parental age, environmental toxins, and other factors. ${ }^{9-13}$

In addition to influencing their utilization of genetics services, parental etiological attributions may shape a range of other medical decisions. A survey-based study of more than 250 parents of children with ASD concluded that parental perceptions of recurrence risk frequently affected family planning decisions, especially among those who perceived their recurrence risk to be high. ${ }^{11}$ Likewise, population-based studies have found evidence that families commonly engage in "reproductive stoppage" after a child is diagnosed with ASD. ${ }^{14}$ Etiological attributions to nongenetic factors may also influence treatment decisions; concerns about a causal relationship between vaccines and ASD have commonly been given as a reason for vaccine refusal, which has been linked to recent measles outbreaks in the USA. ${ }^{15-17}$ Parental endorsement of food allergies as a cause of ASD has also been associated with use of chelation, a controversial intervention that lacks demonstrated efficacy and carries a risk of death. ${ }^{12,18}$

Collectively, these findings highlight the clinical relevance of parental etiological attributions and underscore the need for research aimed at understanding the factors that influence these beliefs. Studies of cross-cultural variation in ASD perceptions have identified numerous factors - including social norms and religious or spiritual beliefs - that may contribute to cultural differences in parental appraisal of ASD symptoms. ${ }^{19,20}$ In addition, parental etiological beliefs about ASD have been shown to differ according to ethnicity, family structure, and income in the USA. ${ }^{21}$ However, it is not known to what extent differences in child clinical presentation may contribute to variation in parental beliefs about ASD etiology.
The autism spectrum is highly heterogeneous with respect to symptom severity and cognitive functioning, encompassing nonverbal children with severe intellectual impairments along with those who test in the superior range on measures of cognitive ability. Several lines of evidence suggest that, compared to children with either condition alone, children with ASD and co-occurring cognitive impairment present with a distinct clinical profile that includes an earlier age of parent-reported onset and an increased risk for comorbid psychopathology and challenging behaviors. ${ }^{22-24}$ Given the potential for parental etiological attributions to be influenced by these factors, the aim of the present study was to compare the causal beliefs held by the parents of children with ASD and comorbid intellectual disability (ID) and/or developmental delay (DD) to those held by the parents of children with either ASD or ID/DD alone. A better understanding of the determinants of parental beliefs about ASD etiology may facilitate improved communication between genetics professionals and affected families.

\section{Methods}

Data were analyzed from the 2011 Survey of Pathways to Diagnosis and Services ("Pathways"). The Pathways survey was conducted by the Centers for Disease Control and Prevention (CDC) through the National Center for Health Statistics, State and Local Area Integrated Telephone Survey program. This survey was a follow-up to the 2009-2010 National Survey of Children with Special Health Care Needs (NS-CSHCN), a cross-sectional, random-digit-dial telephone survey of the parents and guardians of US children with special health care needs (CSHCN) between the ages of 0 and 17 . A random sample of 6,090 NS-CSHCN respondents who had previously indicated that their child had ever been diagnosed with ASD, ID, and/or DD were selected to be recontacted for the Pathways survey. Those who were successfully contacted were further screened to confirm that the eligible child was between the ages of 6 and 17 at the time of the interview, continued to live with the respondent, and had been diagnosed with one of the abovementioned developmental conditions. The Pathways interview was completed by 4,032 respondents. Weighted estimates derived from the Pathways dataset are representative of the US population of noninstitutionalized CSHCN who have ever been diagnosed with ASD, ID, and/or DD. As a secondary analysis of publicly available, deidentified data, this study was exempt from Institutional Review Board approval.

The present analysis was limited to respondents who indicated that their child had ASD, ID, and/or DD at the 
time of the Pathways interview, based on three questions about whether, to the best of the respondent's knowledge, the child currently had "autism or an autism spectrum disorder," "an intellectual disability or mental retardation," and/or "a developmental delay that affected his/her ability to learn." Respondents who met these criteria were further classified into three condition groups: 1) those whose children currently had ASD, without ID or DD ("ASD-only" $n=492$ ); 2) ASD with ID and/or DD ("ASD+ID/DD" n=913); or 3) ID and/ or DD, without ASD ("ID/DD-only" $n=1,949$ ). Pathways respondents who had previously indicated that their child had Down syndrome were excluded.

Parental beliefs about the etiology of their child's developmental disabilities were assessed based on three questions. Respondents were asked whether they thought that their child's condition was 1) "genetic or hereditary"; 2) "caused by something he/she was exposed to in utero, that is, before he/she was born"; and 3) "caused by something he/she was exposed to after he/she was born." These questions were adapted from the Revised Illness-Perception Questionnaire modified for autism (IPQ-RA). A scale was used for each question, with possible responses of "definitely agree," "somewhat agree," "somewhat disagree," and "definitely disagree." Respondents were also permitted to answer "don't know" to each question. In the present analysis, responses were dichotomized into those who endorsed (answered "definitely agree" or "somewhat agree") or did not endorse (answered "definitely disagree," "somewhat disagree," or “don't know") each of the three causes.

The proportion of respondents that endorsed each of the three etiologic categories (genetics, prenatal environmental exposures, and postnatal environmental exposures) was calculated separately for the ASD-only, ASD+ID/DD, and ID/ DD-only groups. Multivariable logistic regression analyses were used to compare beliefs between groups. Age, sex, race, ethnicity, household income, and household education level were included in the models to control for the potentially confounding effects of these demographic characteristics.

Respondents were further classified based on the combination of responses they provided to the three etiology questions: 1) those who endorsed genetics but did not endorse prenatal or postnatal exposures (hereafter referred to as "predominantly genetic"); 2) those who endorsed prenatal and/or postnatal exposures but did not endorse genetics ("predominantly environmental"); 3) those who endorsed genetics plus prenatal and/or postnatal exposures ("genetic and environmental"); and 4) those who endorsed neither genetics nor environmental exposures ("neither"). Multivari- able logistic regression analyses were used to compare the prevalence of each of these response patterns across the three groups (ASD-only, ASD+ID/DD, and ID/DD-only). All models controlled for demographic characteristics (age, sex, race, ethnicity, household income, household education level).

Further analyses were performed to assess the association between parental beliefs and the child's developmental characteristics. Information about the child's history of regression was obtained through a question about whether, at the time that the parent's concerns began, they felt that some of the child's "speech skills that he/she had already developed were lost." This question was only asked of parents who reported that the child's age at the onset of their concerns was at least 9 months. Rao-Scott $\chi^{2}$ tests were used to compare the proportion of respondents that endorsed each of the three etiologic categories (between those who did and did not report a history of language regression).

\section{Results}

Across all groups, a majority of respondents - including 72\% of the ASD-only group, $57 \%$ of the ASD+ID/DD group, and $67 \%$ of the ID/DD-only group - identified genetic or hereditary factors as a cause of their child's condition (Table 1). By contrast, a minority of respondents in each group endorsed prenatal $(28 \%-33 \%)$ or postnatal $(25 \%-40 \%$; Table 1$)$ environmental exposures. Logistic regression analyses showed that, after adjusting for demographic factors, respondents in the ASD+ID/ DD group were significantly less likely than those in the ASDonly and ID/DD-only groups to attribute their child's condition to genetics/heredity (adjusted odds ratio $[\mathrm{aOR}]=0.6$ for both; Table 2). Although endorsement of postnatal exposures did not differ between the two ASD groups, respondents in both were significantly more likely than those in the ID/DD-only group to attribute their child's condition to exposures after birth; just $25 \%$ of the ID/DD-only group (Table 1) endorsed postnatal exposures, compared to $34 \%$ of the ASD-only group (aOR $=1.7$; Table 2 ) and $40 \%$ of the ASD+ID/DD group (aOR $=2.0$ ). Parental endorsement of prenatal exposures did not differ between condition groups (Table 2).

Similar patterns emerged when respondents were classified according to the combination of responses that they provided to the three etiologic questions in the Pathways survey. Although a significant proportion of respondents in each group attributed their child's condition to both genetic factors and environmental exposures (29\%-32\%; Table 1), many appeared to favor one over the other, endorsing either genetics/heredity but not environmental exposures ("predominantly genetic"), or exposures but not genetics/ 
Table I Prevalence of etiological attributions by condition group

\begin{tabular}{|c|c|c|c|c|c|c|c|}
\hline \multirow{2}{*}{$\begin{array}{l}\text { Condition } \\
\text { group }\end{array}$} & \multicolumn{2}{|l|}{ Genetic } & \multicolumn{3}{|l|}{ Environmental } & \multirow{2}{*}{$\begin{array}{l}\text { Both } \\
\text { genetic and } \\
\text { environmental } \\
\text { (\%) }\end{array}$} & \multirow{2}{*}{$\begin{array}{l}\text { Neither } \\
\text { (\%) }\end{array}$} \\
\hline & $\begin{array}{l}\text { Any genetic } \\
\text { (\%) }\end{array}$ & $\begin{array}{l}\text { Predominantly } \\
\text { genetic } \\
\text { (\%) }\end{array}$ & $\begin{array}{l}\text { Any prenatal } \\
\text { exposure } \\
\text { (\%) }\end{array}$ & $\begin{array}{l}\text { Any postnatal } \\
\text { exposure } \\
(\%)\end{array}$ & $\begin{array}{l}\text { Predominantly } \\
\text { environmental } \\
\text { (\%) }\end{array}$ & & \\
\hline ASD+ID/DD & $56.8 \pm 2.9$ & $25.3 \pm 2.4$ & $33.0 \pm 2.7$ & $39.8 \pm 2.9$ & $25.4 \pm 2.5$ & $31.5 \pm 2.8$ & $17.6 \pm 2.2$ \\
\hline ASD-only & $71.8 \pm 3.7$ & $40.7 \pm 4.1$ & $28.1 \pm 3.9$ & $33.9 \pm 3.9$ & $15.4 \pm 3.1$ & $31.0 \pm 3.7$ & $12.8 \pm 2.6$ \\
\hline ID/DD-only & $66.5 \pm 2.1$ & $37.4 \pm 2.2$ & $31.6 \pm 2.0$ & $24.6 \pm 2.1$ & $15.8 \pm 1.4$ & $28.7 \pm 2.2$ & $17.6 \pm 1.9$ \\
\hline
\end{tabular}

Notes: Respondents were asked to indicate the extent to which they believed that genetics/heredity, prenatal exposures, and postnatal exposures had contributed to their child's condition. The "Any genetic" column shows the proportion of respondents that endorsed (answered "definitely" or "somewhat" agree) genetics/heredity; "Predominantly genetic" column shows the proportion that endorsed genetics but not environmental exposures; "Any prenatal" and "Any postnatal" columns show the proportion that endorsed prenatal exposures and postnatal exposures, respectively; "Predominantly environmental" column shows the proportion that endorsed environmental exposures but not genetics; "Both" column shows the proportion that endorsed genetics and environmental exposures; "Neither" column shows the proportion that did not endorse any of the three causes.

Abbreviations: ASD, autism spectrum disorders; DD, developmental delay; ID, intellectual disability.

Table 2 Comparison of etiological attributions by condition group

\begin{tabular}{|c|c|c|c|c|c|c|c|}
\hline \multirow{2}{*}{$\begin{array}{l}\text { Condition } \\
\text { group }\end{array}$} & \multicolumn{2}{|l|}{ Genetic } & \multicolumn{3}{|l|}{ Environmental } & \multirow{2}{*}{$\begin{array}{l}\text { Both } \\
\text { genetic and } \\
\text { environmental } \\
\text { aOR }(95 \% \mathrm{Cl})\end{array}$} & \multirow[t]{2}{*}{ Neither } \\
\hline & $\begin{array}{l}\text { Any genetic } \\
\text { aOR }(95 \% \mathrm{Cl})\end{array}$ & $\begin{array}{l}\text { Predominantly } \\
\text { genetic } \\
\text { aOR }(95 \% \mathrm{Cl})\end{array}$ & $\begin{array}{l}\text { Any prenatal } \\
\text { exposure } \\
\text { aOR }(95 \% \mathrm{Cl})\end{array}$ & $\begin{array}{l}\text { Any postnatal } \\
\text { exposure } \\
\text { aOR }(95 \% \mathrm{Cl})\end{array}$ & $\begin{array}{l}\text { Predominantly } \\
\text { environmental } \\
\text { aOR }(95 \% \mathrm{Cl})\end{array}$ & & \\
\hline $\begin{array}{l}\text { ASD+ID/DD vs } \\
\text { ASD-only }\end{array}$ & $0.6(0.4-0.9)^{*}$ & $0.5(0.3-0.8)^{*}$ & $1.3(0.8-2.0)$ & $1.2(0.8-1.9)$ & $1.8(1.0-3.1)^{*}$ & I.I (0.7-I.7) & I.4 (0.8-2.5) \\
\hline $\begin{array}{l}\text { ASD+ID/DD vs } \\
\text { ID/DD-only }\end{array}$ & $0.6(0.5-0.9)^{*}$ & $0.5(0.4-0.7)^{*}$ & I.I (0.8-1.5) & $2.0(1.4-3.0)^{*}$ & $1.7(1.2-2.5)^{*}$ & $1.2(0.8-1.8)$ & I.I $(0.7-1.7)$ \\
\hline $\begin{array}{l}\text { ASD-only } \\
\text { vs } \\
\text { ID/DD-only }\end{array}$ & $1.2(0.8-1.8)$ & $1.0(0.7-1.5)$ & $0.9(0.5-1.3)$ & $1.7(\mathrm{I} . \mathrm{I}-2.6)^{*}$ & $1.0(0.6-1.7)$ & I.I (0.7-I.7) & $0.8(0.5-1.4)$ \\
\hline
\end{tabular}

Notes: Respondents were asked to indicate the extent to which they believed that genetics/heredity, prenatal exposures, and postnatal exposures had contributed to their child's condition. Parental endorsement of genetics/heredity, prenatal exposures, and postnatal exposures were compared across groups. Multivariable logistic regression analyses controlled for age, sex, race, ethnicity, household income level, and household education level. *Significance, set at the $p<0.05$ level.

Abbreviations: aOR, adjusted odds ratio; ASD, autism spectrum disorders; Cl, confidence interval; DD, developmental delay; ID, intellectual disability.

heredity ("predominantly environmental"). However, the distribution of respondents between the "predominantly genetic" and "predominantly environmental" response categories differed among the three condition groups. In the ASD-only and ID/DD-only groups, the "predominantly genetic" response pattern was more common (ASD-only: 41\%; ID/DD-only: 37\%; Table 1) than the "predominantly environmental" one (ASD-only: 15\%; ID/DD-only: 16\%). In the ASD+ID/DD group, by contrast, respondents were more evenly split, with roughly equal percentages of respondents falling into the "predominantly genetic" and "predominantly environmental" categories ( $25 \%$ each; Table 1$)$. Multivariable logistic regression analyses showed that, compared to those in either of the other condition groups, respondents in the ASD+ID/DD group were significantly less likely to hold "predominantly genetic" beliefs (ASD-only: aOR $=0.5$; ID/ DD-only: aOR $=0.5$; Table 2 ) and significantly more likely to hold "predominantly environmental" beliefs (ASD-only: aOR=1.8; ID/DD-only: aOR=1.7).
Within each group, Rao-Scott $\chi^{2}$ tests were performed to examine the relationship between parental beliefs and parent report of language regression. In the ASD-only and ID/DD-only groups, no associations were identified between regression history and parental beliefs (Table 3). However, in the ASD+ID/DD group, respondents who reported that their child had a history of regression were significantly less likely to endorse genetics $(p=0.006)$ and significantly more likely to endorse postnatal exposures $(p<0.001)$ as contributors to their child's developmental disabilities, compared to those who reported no history of regression (Table 3 ).

\section{Discussion}

Numerous studies have documented the wide range of genetic and nongenetic factors to which parents commonly attribute their child's ASD. Although the diversity in these etiological explanations rivals the heterogeneity of the autism spectrum itself, little is known about the factors that influence these beliefs, and it is unclear whether parental etiological attri- 
Table 3 Association between history of language regression and parental beliefs

\begin{tabular}{|c|c|c|c|c|c|c|c|c|c|}
\hline \multirow{2}{*}{$\begin{array}{l}\text { Condition } \\
\text { group }\end{array}$} & \multicolumn{3}{|l|}{ Genetic } & \multicolumn{3}{|l|}{ Prenatal } & \multicolumn{3}{|l|}{ Postnatal } \\
\hline & Reg. (\%) & Non-reg. (\%) & $p$-value & Reg. (\%) & Non-reg. (\%) & $p$-value & Reg. (\%) & Non-reg. (\%) & $p$-value \\
\hline ASD-only & $63.2 \pm 9.0$ & $72.9 \pm 4.5$ & 0.317 & $34.1 \pm 9.1$ & $17.4 \pm 3.9$ & 0.065 & $45.5 \pm 10.1$ & $26.4 \pm 4.2$ & 0.070 \\
\hline ASD+ID/DD & $48.2 \pm 5.0$ & $66.6 \pm 4.1$ & $0.006 *$ & $32.3 \pm 4.8$ & $31.6 \pm 4.0$ & 0.907 & $51.2 \pm 5.0$ & $28.9 \pm 3.8$ & $<0.00 I^{*}$ \\
\hline ID/DD-only & $71.8 \pm 4.0$ & $69.5 \pm 3.0$ & 0.649 & $31.8 \pm 4.2$ & $31.1 \pm 2.8$ & 0.897 & $23.8 \pm 3.6$ & $22.8 \pm 2.5$ & 0.816 \\
\hline
\end{tabular}

Notes: Within each condition group, $\chi^{2}$ tests were used to compare rates of endorsement of each etiologic category (genetics, prenatal exposures, postnatal exposures) between respondents who reported that their child had a history of language regression and those who reported no regression. $*$ Significance, set at the $p<0.05$ level.

Abbreviations: ASD, autism spectrum disorders; DD, developmental delay; ID, intellectual disability; reg, regression; non-reg, no regression.

butions differ according to the child's cognitive status. The aim of the present study was to use data from a nationally representative sample of more than 3,000 respondents to compare the etiologic beliefs held by the parents of children with ASD and comorbid ID and/or DD (ASD+ID/DD) to those held by the parents of children with either condition alone (ASD-only or ID/DD-only).

A majority of respondents in the Pathways sample believed that their child's condition had a genetic or hereditary basis. Among the parents of children with ASD, the proportion of respondents that endorsed genetics/heredity was $57 \%$ for the ASD+ID/DD group and $72 \%$ for the ASDonly group. Additionally, more than one-fourth (29\%-32\%) of each condition group attributed their child's condition to both genetics and environmental (prenatal and/or postnatal) exposures. These estimates are reasonably consistent with the findings of a 2009 study that reported that $73 \%$ of their sample attributed their child's ASD to genetic influences. ${ }^{11}$ However, the results of the present analysis diverge from the findings of a number of other studies, including one that reported that just $26 \%$ of respondents believed that their child's ASD had a genetic basis. ${ }^{10}$ These discrepancies may be due in part to differences in respondent characteristics; whereas the prior studies utilized relatively small convenience samples, the Pathways study was designed to be nationally representative.

When the three condition groups were compared, the parents of children with ASD and comorbid intellectual/DDs showed a greater tendency to attribute their child's condition to nongenetic factors than the parents of children with either condition alone. After controlling for demographic factors, parents in the ASD+ID/DD group were significantly less likely to attribute their child's condition to genetics/heredity than those in either the ASD-only or ID/DD-only groups. Moreover, when respondents were classified according to the combination of responses they provided to the three etiology questions in the Pathways survey, those in the ASD+ID/DD group were significantly more likely to hold "predominantly environmental" beliefs, and less likely to hold "predominantly genetic" beliefs, than their counterparts in the other condition groups.

Additional analyses identified a subset of respondents in the ASD+ID/DD group that were especially likely to subscribe to nongenetic, external explanations for their child's disabilities. Among the parents of children with ASD+ID/DD, those who reported that their child had a history of language regression were significantly more likely to endorse postnatal exposures as a contributor to their child's condition, and less likely to endorse genetics, than those who indicated that their child had not regressed. These findings are consistent with the results of a 2005 survey that compared etiologic beliefs between parents who reported that their child had always had symptoms of ASD ("congenital") and those who indicated that their child had regressed after a period of normal development ("regressive"). ${ }^{25}$ In that study, parents in the "congenital" group were more likely to view their child's condition as genetic, whereas those in the "regressive" group were more likely to attribute their child's ASD to external factors. Although the Pathways survey did not directly assess the extent to which beliefs influenced parental decision-making in this sample, a number of controversial practices for preventing or treating ASD - such as chelation, withholding of immunizations, antibiotics, antiviral agents, and high-dose vitamins - are predicated on the belief that external factors can trigger or worsen the symptoms of ASD. Thus, future studies should aim to characterize the relationship between parental etiological attributions and use of these alternative therapies.

Although the use of the large, nationally representative Pathways sample was a major strength of the present analysis, the survey-based design of this study was also associated with a few limitations. The Pathways interview asked parents to rate the extent to which they believed that three broad categories of etiologic factors (genetics/heredity, prenatal 
exposures, and postnatal exposures) had contributed to their child's condition. A drawback of this format is that it did not permit assessment of the prevalence of specific etiologic attributions. Parents who endorsed postnatal exposures, for example, may have attributed their child's condition to factors as diverse as vaccines, heavy metals, infections, medications, or other substances. The format of this survey also precluded assessment of etiologic beliefs that did not fall into any of the three categories encompassed by the Pathways questions. Notably, $13 \%-18 \%$ of each group analyzed in this study did not endorse any of the three etiologic categories. Previous studies based on open-ended questions have identified a number of parental etiologic attributions that were not addressed in the Pathways survey, including religious or superstitious factors, parental age, and other causes. ${ }^{20}$

Another limitation of the present study is that the Pathways survey relied on parent-reported diagnoses that were not independently verified. Recent records-based epidemiological studies conducted by the CDC have estimated that $31 \%-41 \%$ of children with ASD have an IQ consistent with ID ( $\leq 70)$, with an additional $21 \%-24 \%$ falling in the "Borderline" (71-85) range. ${ }^{26-28}$ By contrast, just $21 \%$ of children with ASD in the Pathways sample were reported to have ID, while $44 \%$ were reported to have ASD+DD without ID. This suggests that ID may have been underreported in this sample, and that some of the children who were identified as having ASD+DD may have had cognitive impairment consistent with ID even if the parents reported otherwise. Thus, the present study grouped children with ASD-only separately from those with ASD plus ID and/or DD (ASD+ID/DD) to preserve the distinction between children with normal intellectual functioning and those with some degree of cognitive impairment. This classification is supported by the fact that, among children with ASD in the Pathways sample, the prevalence of comorbid ID and/or DD (65\%) roughly corresponds to the total percent of children in the records-based epidemiological studies $(54 \%-62 \%)$ that tested below the average range on measures of IQ $(\leq 85) \cdot{ }^{26-28}$ However, the ASD+ID/DD group was likely clinically heterogeneous, and the inability to corroborate parent-reported diagnoses with validated measures of functioning represents a significant limitation of the present study.

\section{Conclusion}

Notwithstanding the limitations discussed above, the findings of this study are highly relevant to clinicians who provide care to children with developmental disabilities and their families. Genetics professionals, in combination with other clinicians involved in the diagnostic process
- including psychiatrists, developmental pediatricians, neurologists, and primary care providers - play a vital role in educating parents about etiology and helping them to access recommended genetics services for their children. This study showed that the parents of children with ASD and comorbid ID/DD were significantly less likely than the parents of children with either condition alone to attribute their child's condition to genetic or hereditary factors, especially if the child had a history of language regression. Given that parental etiological attributions may influence their utilization of numerous services for their children, including genetic testing, an awareness of the factors that influence parental etiological beliefs may allow clinicians to provide more individualized counseling to affected families. Overall, it is hoped that a better understanding of the determinants and consequences of parental etiological beliefs will facilitate improved access to genetics services in this population.

\section{Acknowledgment}

The authors would like to thank Dr. Sarah Keim for her assistance with reviewing the statistical analyses.

\section{Author contributions}

All authors contributed to data analysis, drafting or revising the article, gave final approval of the version to be published, and agree to be accountable for all aspects of the work.

\section{Disclosure}

The authors report no conflicts of interest in this work.

\section{References}

1. Zablotsky B, Black L, Maenner M, Schieve L, Blumberg S. Estimated prevalence of autism and other developmental disabilities following questionnaire changes in the 2014 National Health Interview Survey. Natl Health Stat Rep. 2015;13:1-21.

2. El-Fishawy P, State MW. The genetics of autism: key issues, recent findings, and clinical implications. Psychiatr Clin North Am. 2010;33: $83-105$.

3. Betancur C. Etiological heterogeneity in autism spectrum disorders: more than 100 genetic and genomic disorders and still counting. Brain Res. 2011;1380:42-77.

4. Schaefer GB, Mendelsohn NJ. Clinical genetics evaluation in identifying the etiology of autism spectrum disorders: 2013 guideline revisions. Genet Med. 2013;15:399-407.

5. Cuccaro ML, Czape K, Alessandri M, et al. Genetic testing and corresponding services among individuals with autism spectrum disorder (ASD). Am J Med Genet. 2014;164(10):2592-2600.

6. Kiely B, Vettam S, Adesman A. Utilization of genetic testing among children with developmental disabilities in the United States. Appl Clin Genet. 2016;9:93-100.

7. Chen LS, Xu L, Huang TY, Dhar SU. Autism genetic testing: a qualitative study of awareness, attitudes, and experiences among parents of children with autism spectrum disorders. Genet Med. 2013;15(4):274. 
8. Reiff M, Bugos E, Giarelli E, et al. "Set in Stone" or "Ray of Hope": parents' beliefs about cause and prognosis after Genomic testing of children diagnosed with ASD. JAutism Dev Disord. 2017;47(5):1453-1463.

9. Mercer L, Creighton S, Holden J, Lewis M. Parental perspectives on the causes of an autism spectrum disorder in their children. $J$ Genet Couns. 2006;15:41-50.

10. Harrington JW, Patrick PA, Edwards KS, Brand DA. Parental beliefs about autism implications for the treating physician. Autism. 2006;10:452-462.

11. Selkirk CG, Veach PM, Lian F, Schimmenti L, LeRoy BS. Parents' perceptions of autism spectrum disorder etiology and recurrence risk and effects of their perceptions on family planning: recommendations for genetic counselors. J Genet Couns. 2009;18:507-519.

12. Dardennes RM, Al Anbar NN, Prado-Netto A, Kaye K, Contejean Y, Al Anbar NN. Treating the cause of illness rather than the symptoms: parental causal beliefs and treatment choices in autism spectrum disorder. Res Dev Disabil. 2011;32:1137-1146.

13. Al Anbar NN, Dardennes RM, Prado-Netto A, Kaye K, Contejean Y. Treatment choices in autism spectrum disorder: the role of parental illness perceptions. Res Dev Disabil. 2010;31:817-828.

14. Hoffmann TJ, Windham GC, Anderson M, Croen LA, Grether JK, Risch N. Evidence of reproductive stoppage in families with autism spectrum disorder: a large, population-based cohort study. JAMA Psychiatry. 2014;71:943-951.

15. Gahr P, DeVries AS, Wallace G, et al. An outbreak of measles in an undervaccinated community. Pediatrics. 2014;134:e220-e228.

16. Sugerman DE, Barskey AE, Delea MG, et al. Measles outbreak in a highly vaccinated population, San Diego, 2008: role of the intentionally undervaccinated. Pediatrics. 2010;125:747-755.

17. Rosenberg RE, Law JK, Anderson C, Samango-Sprouse C, Law PA Survey of vaccine beliefs and practices among families affected by autism spectrum disorders. Clin Pediatr. 2013;52(9):871-874.
18. Baxter AJ, Krenzelok EP. Pediatric fatality secondary to EDTA chelation. Clin Toxicol. 2008;46:1083-1084.

19. Bernier R, Mao A, Yen J. Psychopathology, families, and culture: autism. Child Adolesc Psychiatr Clin N Am. 2010;19(4):855-867.

20. Jegatheesan B, Miller PJ, Fowler SA. Autism from a religious perspective: a study of parental beliefs in South Asian Muslim immigrant families. Focus Autism Other Dev Disabl. 2010;25:98-109.

21. Zuckerman KE, Lindly OJ, Sinche B. Parent beliefs about the causes of learning and developmental problems among children with autism spectrum disorder: results from a national survey. Am J Intellect Dev Disabil. 2016;121:432-447.

22. De Giacomo A, Fombonne E. Parental recognition of developmental abnormalities in autism. Eur Child Adolesc Psychiatry. 1998;7(3): $131-136$

23. Zuckerman KE, Lindly OJ, Sinche BK. Parental concerns, provider response, and timeliness of autism spectrum disorder diagnosis. J Pediatr. 2015;166(6):1431-1439.

24. Matson JL, Shoemaker M. Intellectual disability and its relationship to autism spectrum disorders. Res Dev Dis. 2009;30(6) 1107-1114

25. Goin-Kochel RP, Myers BJ. Congenital versus regressive onset of autism spectrum disorders: parents' beliefs about causes. Focus Autism Other Dev Disabl. 2005;20:169-179.

26. CDC. Prevalence of autism spectrum disorders - autism and developmental disabilities monitoring network, United States, 2006. MMWR Surveill Summ. 2009;58(10):1-24.

27. CDC. Prevalence of autism spectrum disorders - autism and developmental disabilities monitoring network, 14 Sites, United States, 2008 MMWR Surveill Summ. 2012;61(3):1-9.

28. CDC. Prevalence of autism spectrum disorder among children aged 8 years - autism and developmental disabilities monitoring network, 11 sites, United States, 2010. MMWR Surveill Summ. 2014;63(2):1-21.
The Application of Clinical Genetics

\section{Publish your work in this journal}

The Application of Clinical Genetics is an international, peer-reviewed open access journal that welcomes laboratory and clinical findings in the field of human genetics. Specific topics include: Population genetics; Functional genetics; Natural history of genetic disease; Management of genetic disease; Mechanisms of genetic disease; Counselling and ethical

\section{Dovepress}

issues; Animal models; Pharmacogenetics; Prenatal diagnosis; Dysmorphology. The manuscript management system is completely online and includes a very quick and fair peer-review system, which is all easy to use. Visit http://www.dovepress.com/testimonials.php to read real quotes from published authors. 